\title{
FUNGOS MICORRÍZICOS ARBUSCULARES (GLOMALES, ZYGOMYCOTA) NA ILHA DOS EUCALIPTOS, REPRESA DO GUARAPIRANGA, SÃO PAULO, SP'
}

\author{
Simone de Paula Gomes ${ }^{2}$ \\ Sandra Farto Botelho Trufem ${ }^{2}$
}

Recebido em 22/01/1999. Aceito em 27/04/1999

\begin{abstract}
RESUMO - (Fungos micorrízicos arbusculares (Glomales, Zygomycota) na Ilha dos Eucaliptos, Represa do Guarapiranga, São Paulo, SP). Com o objetivo de se conhecer a micota de fungos micorrízicos arbusculares (FMA, Glomales, Zygomycota), coletou-se amostras de solo rizosférico de plantas instaladas em três áreas (plantio de eucaliptos, mata e transição entre ambas) em três épocas (março/1996, época chuvosa e final de verão; junho/1996, época de inverno; janeiro/1997, início das chuvas e do verão) na Illha dos Eucaliptos (cerca de $323.500 \mathrm{~m}^{2}$ ), na Represa do Guarapiranga, São Paulo, SP. O solo foi processado para a separação dos esporos de FMA segundo a técnica do peneiramento e decantação em via úmida, seguindo-se centrifugação em solução de sacarose $50 \%(\mathrm{~V}: \mathrm{V})$. Os esporos isolados foram preparados em lâminas permanentes com resina PVL. Foram constatados 21 táxons de FMA: Acaulospora excavata Ingleby, Walker \& Mason, Acaulospora foveata Trappe \& Janos, Acaulospora longula Spain \& Schenck, Acaulospora mellea Spain \& Schenck, Acaulospora morrowiae Spain \& Schenck, Acaulospora spinosa Walker \& Trappe, Acaulospora sp. 1, Acaulospora sp. 2, Acaulospora sp. 3, Acaulospora sp. 4, Entrophospora colombiana Spain \& Schenck, Gigaspora gigantea Nicol. \& Gerd., Gigaspora ramisporophora Spain, Sieverding \& Schenck, Glomus aggregatum (Schenck \& Smith) Koske, Glomus etunicatum Becker \& Gerd., Glomus geosporum (Nicol. \& Gerd.) Walker, Glomus invermayum Hall, Glomus macrocarpum Tul. \& Tul., Glomus multisubstensum Mukerjii, Bhattacharjee \& Tewari, Scutellospora aff. biornata Spain, Sieverding \& Toro e Scutellospora erythropa Koske \& Walker. A aplicação do índice de similaridade de Sörensen entre populações de FMA nas diferentes áreas e as épocas de coletas mostrou valores de 64 a 90\%, sugerindo não haver seleção dos FMA mediada por esses fatores.
\end{abstract}

Palavras-chaves - FMA, diversidade, similaridade

ABSTRACT - (Arbuscular mycorrhizal fungi (Glomales, Zygomycota) from "Ilha dos Eucaliptos", Guarapiranga Reservoir, São Paulo, SP, Brazil). With the aim of verifying the occurence of arbuscular mycorrhizal fungi AMF, Glomales, Zygomycota), rhizosphere soil samples were collected in three areas (eucaliptus reforestation, forest and intersection) and on three occasions (March/1996, rainy and warm; June/1996, dry and cold; and January/1997, the beginning of both the rainy season and the summer) on the "Ilha dos Eucaliptos" (ca. $323.500 \mathrm{~m}^{2}$ ) in the Guarapiranga Reservoir, São Paulo, SP, Brazil. Soil samples were processed by the wet sieving technique, followed by sucrose centrifugation ( $50 \% \mathrm{~V}: \mathrm{V})$. The isolated spores were prepared in vouchers, with polivynil alchool lactofenol resin (PVL). We observed 21 taxa of AMF: Acaulospora excavata Ingleby, Walker \& Mason, Acaulospora foveata Trappe \& Janos,

1 Trabalho de Monografia da primeira Autora; apresentado no $49^{\circ} \mathrm{CNB}$, Salvador, BA, no concurso Prêmio Verde

2 Instituto de Botânica, C. Postal 4005, CEP 01061-970, São Paulo, SP, Brasil. e-mail: strufem@cyberspace.com.br 
Acaulospora longula Spain \& Schenck, Acaulospora mellea Spain \& Schenck, Acaulospora morrowiae Spain \& Schenck, Acaulospora spinosa Walker \& Trappe, Acaulospora sp. 1, Acaulospora sp. 2, Acaulospora sp. 3, Acaulospora sp. 4, Entrophospora colombiana Spain \& Schenck, Gigaspora gigantea Nicol. \& Gerd., Gigaspora ramisporophora Spain, Sieverding \& Schenck, Glomus aggregatum (Schenck \& Smith) Koske, Glomus etunicatum Becker \& Gerd., Glomus geosporum (Nicol. \& Gerd.) Walker, Glomus invermayum Hall, Glomus macrocarpum Tul. \& Tul., Glomus multisubstensun Mukerjii, Bhattacharjee \& Tewari, Scutellospora aff. biomata Spain, Sieverding \& Toro, and Scutellospora erythropa Koske \& Walker. The Sörensen Index showed $64 \%$ to $90 \%$ of similarity between the areas and the collectings, this suggesting the absence of influence of these kinds of parameters on the mycota.

Key words - AMF, diversity, similarity

\section{Introdução}

A bacia do Guarapiranga abrange os municípios de São Paulo, Embu, EmbuGuaçu e Itapecerica da Serra e atualmente contribui com vazão de $9,5 \mathrm{~m}^{3} /$ segundo, atendendo aproximadamente a três milhões de habitantes e suprindo cerca de $20 \%$ do abastecimento de água da região metropolitana de São Paulo (Melo 1994).

A Ilha dos Eucaliptos, também conhecida por Ilha Cumbica, está situada no compartimento central da Represa do Guarapiranga, sendo a maior das quatro ilhas nela presentes, com área aproximada de $323.500 \mathrm{~m}^{2}$ (Wellbaum 1996).

As micorrizas arbusculares consistem em associações simbióticas não antagônicas estabelecidas entre raízes de plantas fanerogâmicas e pteridófitas com fungos Glomales (Zygomycota), estes com cerca de 160 espécies descritas. São caracterizadas pela formação, no córtex da planta hospedeira, de vesículas e arbúsculos, que são estruturas relacionadas com a reserva de nutrientes e troca de metabólitos, respectivamente. As micorrizas arbusculares apresentam ocorrência mais generalizada em regiões tropicais, estimando-se que 90\% das plantas fanerogâmicas apresentem este tipo de associação (Morton \& Benny 1990).

Os fungos micorrízicos arbusculares (FMA) apresentam micélio extra- radicular e exploram mais eficientemente o solo, por atingir áreas onde as raízes não alcançam. Assim, mesmo em solos pobres em nutrientes, a planta consegue desenvolver-se satisfatoriamente, graças à água e aos elementos minerais (principalmente fósforo, mas também zinco, potássio, cálcio, manganês, níquel, cobre, enxofre e ferro) absorvidos pelo fungo. Em troca, a planta hospedeira cede fotossintatos ao fungo (Cooper \& Tinker 1978). Desta forma, a planta hospedeira apresenta crescimento acelerado e desenvolve-se com maior vigor (Francis $e t$ al. 1986), o que lhe confere maior resistência ao ataque por microrganismos do solo, como outros fungos, bactérias e nematóides (Zambolim \& Siqueira 1985).

Em áreas cujo solo apresenta deficiência nutricional, os FMA podem beneficiar o cultivo de plantas agrícolas, maximizando a produção e diminuindo as demandas de fertilizantes e biocidas bem como atuam em áreas degradadas e locais erodidos, promovendo o aumento da cobertura vegetal facilitando sua recuperação (Zambolim \& Siqueira 1985).

Considerando que na Ilha dos Eucaliptos estão sendo concentrados estudos em diferentes áreas do conhecimento, com levantamentos em plantas superiores (Begovacz 1995), insetos (Melo 1994) e diversos grupos de fungos (Welbaum 96; Gomes 1997), este trabalho teve por objetivo conhecer a diversidade de FMA na ilha, em três áreas diferenciadas pela composição fitossociológica (eucaliptos, mata secundária e transição) e em três épocas com características climatológicas distintas. 


\section{Material e métodos}

Caracterização da área estudada - Os estudos foram conduzidos na Ilha dos Eucaliptos, que está localizada na parte central da Represa do Guarapiranga, São Paulo, SP. Apresenta terreno de idade terciária, com solo resultante da decomposição de rochas cristalinas (gnaisse e mica-xistos) e magmáticas (granito). Os índices pluviométricos anuais variam de 1.350 a $2.000 \mathrm{~mm}$, com concentrações de chuvas no verão, e seca no inverno e temperaturas médias anuais entre 14 e $26^{\circ} \mathrm{C}$. vegetação da ilha consiste em uma área reflorestada por eucaliptos, uma área com mata secundária e remanescentes de Mata Atlântica, e uma área de transição entre ambas ( Begovacz 1995; Wellbaum 1996).

Épocas de coleta - As coletas foram realizadas nos meses de março e junho/1996 e janeiro/1997. Essas três épocas foram determinadas em função das diferenças climáticas: janeiro é caracterizado por ser úmido e quente; junho, por ser frio e seco, e março por apresentar situação intermediária entre as duas anteriores.

Coletas de solo - As coletas de solo rizosférico foram realizadas nas três áreas distintas: área reflorestada por eucaliptos, com aproximandamente $182.000 \mathrm{~m}^{2}$, faixa intermediária, a qual foi designada transição, com cerca de $40.500 \mathrm{~m}^{2}$ e mata secundária, com $101.000 \mathrm{~m}^{2}$. Foram escolhidos, aleatoriamente, cinco pontos em cada área para serem realizadas as coletas de solo. Em cada ponto determinado foram retiradas três sub-amostras de solo rizosférico, que após homogeneizadas, formaram uma amostra composta. Assim, em cada área foram coletadas cinco amostras compostas, totalizando 15 amostras ao final de cada coleta. As coletas foram feitas a profundidades de 5 a $20 \mathrm{~cm}$, sempre com atenção em atingir a rizosfera da planta escolhida. As amostras foram acondicionadas em sacos plásticos identificados. O material coletado foi mantido sob refrigeração $\left(4-8^{\circ} \mathrm{C}\right)$ até processamento. Amostras de solo das três áreas foram encaminhadas para análise química e físico-química (Tab.1). Foram registradas temperatura e umidade do solo em cada ocasião de coleta (Tab. 2).

Tabela 1. Análise química e físico-química dos solos estudados, na Ilha dos Eucaliptos, em coleta de janeiro/ 1997.

\begin{tabular}{|c|c|c|c|c|c|c|c|c|c|c|c|c|c|c|}
\hline & \multirow{2}{*}{$\begin{array}{c}\mathrm{P} \\
(\mathrm{ppm})\end{array}$} & \multirow{2}{*}{$\begin{array}{l}\text { M.O. } \\
(\%)\end{array}$} & \multirow[t]{2}{*}{$\mathrm{pH}$} & K & $\mathrm{Ca}$ & $\mathrm{Mg}$ & $\mathrm{H}+\mathrm{Al}$ & \multirow[t]{2}{*}{ SB } & \multirow{2}{*}{\multicolumn{2}{|c|}{ CTC V (\%) }} & \multirow{2}{*}{\multicolumn{4}{|c|}{$\begin{array}{l}\mathrm{Cu} \quad \mathrm{Fe} \\
\mathrm{mg} / \mathrm{dm}^{3}\end{array}$}} \\
\hline & & & & & 1100 & $\mathrm{~cm}^{3}$ & & & & & & & & \\
\hline Eucalipto & 12 & 7 & 3.4 & 0.12 & 0.1 & 0.1 & 22,8 & 0.3 & 23.1 & 1 & 0.57 & 0.5 & 240 & 2.3 \\
\hline Transição & 12 & 6.6 & 3.4 & 0.15 & 0.2 & 0.2 & 22,8 & 0.6 & 23.4 & 2 & 0.53 & 0.5 & 174 & 3.3 \\
\hline Mata & 19 & 7 & 3.2 & 0.15 & 0.2 & 0.2 & 25,3 & 0.6 & 25.9 & 2 & 0.52 & 0.5 & 309 & 2.8 \\
\hline
\end{tabular}

$\bar{M} . \mathrm{O}=$ matéria orgânica; $\mathrm{SB}=$ soma de bases; $\mathrm{CTC}=$ capacidade de troca iônica; $\mathrm{V}=$ saturação de bases.

Tabela 2. Médias da temperatura e teor de umidade dos solos estudados na Ilha dos Eucaliptos, nos meses de março e junho/1996 e janeiro/1997.

\begin{tabular}{lccccccc}
\hline & \multicolumn{3}{c}{ Temperatura $\left({ }^{\circ} \mathrm{C}\right)$} & & \multicolumn{3}{c}{ Teor de Umidade $(\%)$} \\
\cline { 2 - 4 } \cline { 6 - 8 } Período de coleta & $\mathrm{E}$ & $\mathrm{T}$ & $\mathrm{M}$ & & $\mathrm{E}$ & $\mathrm{T}$ & $\mathrm{M}$ \\
\hline março/96 & 20,3 & 19,2 & 19,6 & & 30,7 & 39,4 & 31,7 \\
junho/96 & 17,0 & 17,0 & 17,0 & & 26,0 & 29,1 & 40,3 \\
janeiro/97 & 20,0 & 20,0 & 20,0 & & 35,4 & 29,2 & 32,8 \\
\hline
\end{tabular}

E=eucalipto; $\mathrm{T}=$ transição e $\mathrm{M}=$ mata 
Isolamento e identificação de esporos - Os esporos de FMA foram isolados pela técnica do peneiramento por via úmida a partir de amostras de solo $(100 \mathrm{~g})$ de rizosfera (Gerdemann \& Nicolson 1963), seguindo-se centrifugação do solo em solução de sacarose 50\% (V:V), segundo Daniels \& Skipper (1982). Os esporos obtidos foram separados, contados e dispostos em lâminas com resina de álcool polivinílico e lactofenol (PVL) para a preparação de lâminas semi-permanentes e observações ao microscópio de luz. As identificações foram feitas com a utilização do manual de descrição de FMA de Schenck \& Pérez (1988), além de referências mais recentes para os táxons posteriormente descritos.

Aspectos ecológicos - Aplicou-se o índice de Sörensen (Müller-Dombois \& Ellemberg 1974) às populações de FMA nas três áreas estudadas (Eucalipto, Mata e Transição) e nas três épocas de coletas para a determinação da similaridade existente entre elas. O índice é dado pela fórmula: $\mathrm{IS}=(2 \mathrm{C} / \mathrm{A}+\mathrm{B}) \cdot 100$, onde $\mathrm{A}$ e $\mathrm{B}$ representam o número total de táxons em cada uma das áreas e $\mathrm{C}$ representa o número de táxons comuns ambas.

\section{Resultados e discussão}

Táxons verificados - Foram verificados 21 táxons de FMA, a saber: Acaulospora excavata Ingleby, Walker \& Mason, A. foveata Trappe \& Janos, A. longula Spain \& Schenck, A. mellea Spain \& Schenck, A. morrowiae Spain \& Schenck, A. spinosa Walker \& Trappe, Acaulospora sp. 1, Acaulospora sp. 2, Acaulospora sp. 3, Acaulospora sp. 4, Entrophospora colombiana Spain \& Schenck, Gigaspora gigantea Nicol. \& Gerd., G. ramisporophora Spain, Sieverding \& Schenck, Glomus aggregatum (Schenck \& Smith) Koske, G. etunicatum Becker \& Gerd., G. geosporum (Nicol. \& Gerd.) Walker, G. invermayum Hall, G. macrocarpum Tul. \& Tul., G. multisubstensum Mukerjii, Bhattacharjee \& Tewari, Scutellospora aff. biornata Spain, Sieverding \& Toro e S. erythropa Koske \& Walker.

A ocorrência das espécies de FMA verificadas nas diferentes áreas e nos diferentes períodos de coleta pode ser observada na Tab. 3. Melhor visualização destes dados é apresentada na Fig. 1, que traz o número total de táxons verificados em cada ecossistema, e na Fig. 2, que traz o somatório de táxons verificados nas três áreas, independente da época de coleta.

Estudos sobre microrganismos são importantes para o entendimento de processos básicos ocorrentes na biosfera (Priorities for Microbial Biodiversity Research 1995). A proposta primordial deste trabalho foi a de determinar a diversidade de FMA na Ilha dos Eucaliptos, sendo constatada a ocorrência de elevado número de táxons: 18 na área de eucaliptos, 15 na transição e 17 na mata. Trabalhos sobre diversidade vêm sendo mundialmente enfatizados. Em ecossistemas tropicais, o interesse é grande devido a exuberância em fauna e flora, onde muitas espécies não foram ainda descritas. Neste trabalho, quatro espécies de Acaulospora possivelmente constituem espécies novas. 


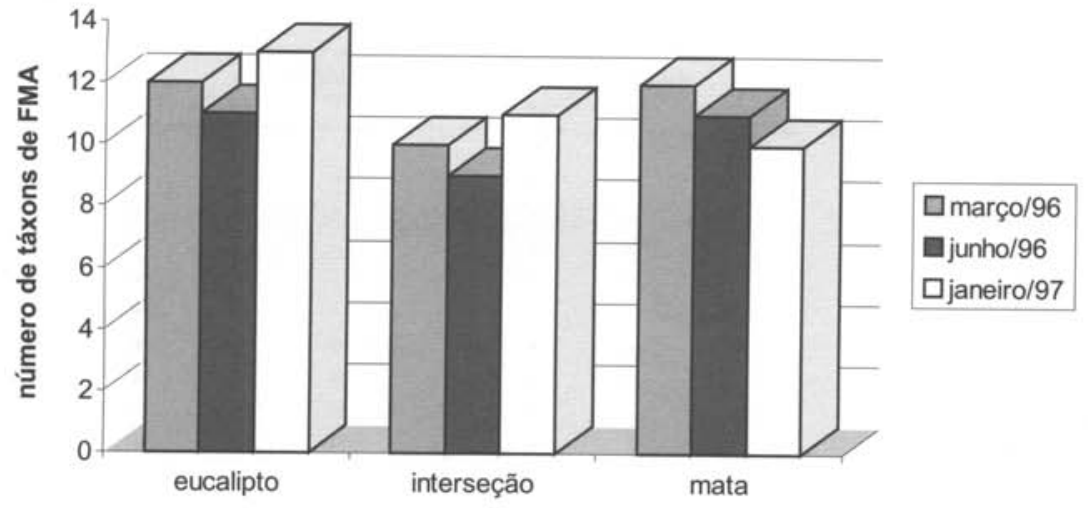

Figura 1. Número total de táxons de FMA verificados nas três épocas de coleta, nas diferentes áreas na Illha dos Eucaliptos

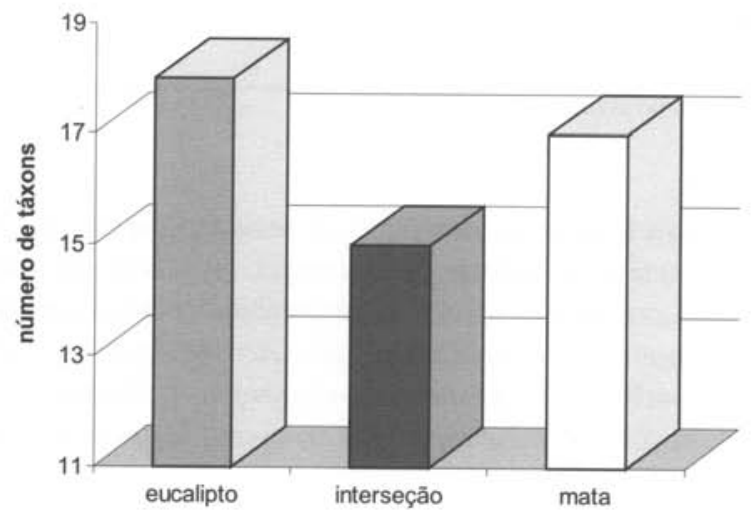

Figura 2. Comparação entre o número total de táxons de FMA encontradas nas três áreas da Ilha dos Eucaliptos.

Comparando-se o número de táxons de FMA (21) verificados na Ilha dos Eucaliptos (Tab. 3) com outros trabalhos em ecossistemas perturbados de Mata Atlântica em São Paulo, constatou-se que Trufem \& Viriato (1990) isolaram 16 táxons e Trufem \& Malatinszky (1995), 23 táxons. Entretanto, quando se comparou os resultados com os de áreas de Mata Atlântica no mesmo Estado, porém não submetidas a impactos ambientais, verificou-se nessas maior diversidade de FMA, com 54 táxons (Trufem 1988). 
Tabela 3. Espécies de FMA verificadas nas áreas estudadas da Ilha dos Eucaliptos, nas três épocas de coleta ( $\mathrm{E}=$ Eucaliptos; $\mathrm{T}=$ Transição; $\mathrm{M}=$ mata).

\begin{tabular}{|c|c|c|c|c|c|c|c|c|c|}
\hline \multirow[b]{2}{*}{ Táxons } & \multicolumn{3}{|c|}{ março/96 } & \multicolumn{3}{|c|}{ junho/96 } & \multicolumn{3}{|c|}{ janeiro/97 } \\
\hline & E & $\mathrm{T}$ & $\mathrm{M}$ & $\mathrm{E}$ & $\mathrm{T}$ & $\mathrm{M}$ & $\mathrm{E}$ & $\mathrm{T}$ & $\mathrm{M}$ \\
\hline Acaulospora excavata & + & $\cdot$ & $\cdot$ & - & - & - & - & - & - \\
\hline A. foveata & + & + & + & + & + & + & + & + & + \\
\hline A. longula & - & - & - & + & - & + & + & - & - \\
\hline A. mellea & - & + & - & - & - & $\cdot$ & + & - & - \\
\hline A. morrowiae & - & - & - & + & - & - & + & + & + \\
\hline A. spinosa & + & - & - & - & - & - & + & + & - \\
\hline Acaulospora sp. 1 & + & + & + & - & + & + & + & + & - \\
\hline Acaulospora sp. 2 & + & + & + & + & + & + & + & + & + \\
\hline Acaulospora sp. 3 & - & - & - & - & - & + & - & - & - \\
\hline Acaulospora sp. 4 & - & - & + & + & + & + & + & + & + \\
\hline Entrophospora colombiana & + & + & + & + & + & + & + & + & - \\
\hline Gigaspora gigantea & + & + & + & + & - & + & - & - & - \\
\hline G. ramisporophora & $\cdot$ & + & + & + & + & - & + & + & + \\
\hline Glomus aggregatum & - & - & - & - & - & + & - & - & - \\
\hline G. etunicatum & + & + & + & - & - & $\cdot$ & - & - & - \\
\hline G. geosporum & - & - & + & - & + & - & - & - & + \\
\hline G. invermayum & + & - & + & - & - & - & - & + & + \\
\hline G. macrocarpum & + & + & + & + & + & + & + & + & + \\
\hline G. multisubstensum & - & - & - & + & - & - & - & - & - \\
\hline Scutellospora aff. biornata & + & - & - & - & - & - & + & - & + \\
\hline Scutellospora erythropa & + & + & + & + & + & + & + & + & + \\
\hline número total de táxons & 12 & 10 & 12 & 11 & 9 & 11 & 13 & 11 & 10 \\
\hline
\end{tabular}

Presença do táxons +; ausência do táxon

Entre as espécies mais freqüentes, destacam-se Acaulospora foveata, Acaulospora sp. 2, Glomus macrocarpum e Scutellospora erytrhopa, presentes em todas as áreas e nos diferentes meses. Algumas espécies foram também abundantes, porém, apresentaram variações na ocorrência por coleta ou por área, como Acaulospora sp. 1, Acaulospora sp. 4, Entrophospora colombiana e Gigaspora ramisporophora, enquanto outros táxons foram esporadicamente encontrados: Acaulospora excavata, Acaulospora sp. 3, Glomus aggregatum e Glomus multisubstensum.

Destes táxons, Acaulospora foveata e Glomus macrocarpum têm sido considerados de comum ocorrência no Estado de São Paulo, como em plantas ornamentais (Trufem et al. 1990; Grandi \& Trufem 1991), em vegetação de Mata Atlântica (Trufem et al. 1990), em culturas de citros (Carrenho 1993) e de amendoim (Bononi et al. 1988).

Bononi \& Trufem (1983) propuseram que a ocorrência generalizada ou limitada de FMA em um dado ecossistema pode representar maior ou menor agressividade ecológica, respectivamente. Na primeira condição ocorrem espécies presentes em grande quantidade de hospedeiros, em todas as épocas do ano, e na segunda, as espécies têm ocorrência esporádica e em plantas específicas.

A diferença no número de espécies de FMA verificado na Ilha, entre os diferentes períodos, não foi significativa, sendo computados 16, 15 e 15 táxons em março e junho/ 1996 e janeiro/1997, respectivamente (Tab. 3). 
Considerando o tipo de vegetação estabelecida nas três áreas estudadas, também não se observou diferenças significativas no número de táxons de FMA nas diferentes épocas de coletas (Fig. 1), a saber: em eucaliptos, 12, 11 e 13 espécies, na área de transição 10,9 e 11 táxons, e na mata 12,11 e 10. Esses dados levam a supor que a população de FMA na Ilha dos Eucaliptos não sofreu influências diretas em sua diversidade tanto em relação à época do ano, como também em função do tipo de vegetação. Abbott \& Gazey (1994), em trabalhos sobre ecologia de FMA, afirmam que diferenças populacionais de FMA podem ser encontradas em tais circunstâncias. Vale ressaltar que estudos referentes à ecologia de FMA são mais abundantes em ambientes temperados do que em tropicais e revelam que o comportamento dos FMA em tais ambientes pode ser diferente (Siqueira \& Franco 1988).

Em relação aos fatores abióticos, sabe-se que os FMA são tolerantes a grandes variações de temperatura, ocorrendo tanto em regiões frias como quentes, embora seja observado que seu crescimento torna-se mais intenso com o aumento da temperatura do solo (Mosse et al. 1981). Em solos tropicais, a temperatura é relativamente constante, de maneira que seu efeito sobre os FMA é menor se comparado a ambientes onde existam oscilações mais amplas.

FMA suportam, de modo geral, amplas variações de $\mathrm{pH}$, preferindo solos ligeiramente ácidos (Siqueira \& Franco 1988). No entanto, Zambolim \& Siqueira (1985) mencionam que espécies de Glomus são comumente encontradas em solos de neutro a alcalinos. Quanto aos gêneros Acaulospora, Gigaspora e Scutellospora, estes mesmos autores afirmam que têm ocorrência em faixa mais ampla de $\mathrm{pH}$, tendendo a ser mais freqüentes em $\mathrm{pH}$ ácidos.

As diferenças verificadas no $\mathrm{pH}$ nas três áreas são pequenas, daí acreditar-se que este elemento não tenha influenciado na ocorrência dos FMA. Também em relação ao $\mathrm{pH}$ tem-se o trabalho de Lima (1993) que menciona experimentos conduzidos na Índia e na Espanha, onde foi constatada acidificação do solo. Embora os eucaliptos tivessem sido introduzidos na Ilha há cerca de cinco anos, quando por ocasião das coletas do presente trabalho o efeito de queda do $\mathrm{pH}$ não foi verificado (Tab. 2), o que traz mais controvérsias em relação ao efeito destas plantas sobre o solo e a microbiota.

FMA não toleram excesso de umidade, que afeta diretamente a solubilidade e disponibilidade de nutrientes do solo. Em solos muito úmidos, ou em ambientes aquáticos, a suscetibilidade das plantas às micorrizas, de modo geral, é menor (Mosse et al. 1981). Entretanto, os valores encontrados na Ilha dos Eucaliptos parecem não afetar negativamente o desempenho dos FMA no ecossistema. A mata apresentou maior umidade em junho/1996 em relação às demais áreas (Tab. 2), podendo este fato estar relacionado com o tipo de vegetação mais densa, que expõe menos o solo, além da retenção de água pela serapilheira.

Quando se considera os elementos minerais do solo nas três diferentes áreas (Tab. 1), percebe-se que o teor de fósforo nas áreas de eucalipto e transição foi semelhante (12ppm), enquanto no solo da mata, o teor foi superior (19 ppm). Os demais elementos encontram-se com valores semelhantes nas diferentes áreas. O maior nível de fósforo na mata pode ser explicado pela vegetação mais abundante e diversificada, que confere melhor equilíbrio ao ambiente, em termos de ciclagem de nutrientes (Malavolta 1980). 
No entanto, aparentemente, esses parâmetros não afetaram a ocorrência nas diferentes áreas, como será discutido a seguir.

A aplicação do índice de similaridade de Sörensen (IS) a partir dos dados da Tab. 3, permitiu comparar a diversidade de espécies de FMA entre as três áreas estudadas, bem como nos diferentes momentos de amostragem (Tab. 4).

Tabela 4. Similaridade da micota verificada pela aplicação do Indice de Sörensen (IS) nas diferentes áreas (Eucalipto, Transição e Mata) e em relação às diferentes épocas de coletas (março/1996, junho/1996 e janeiro/1997).

\begin{tabular}{lc}
\hline Áreas e épocas comparadas & IS (\%) \\
\hline Eucalipto X Transição & 85 \\
Transição X Mata & 81 \\
Mata X Eucalipto & 80 \\
Março X Junho & 64 \\
Junho X Janeiro & 73 \\
Janeiro X Março & 90 \\
\hline
\end{tabular}

A análise destes valores indica que, de fato, a similaridade da composição específica de FMA nas três áreas é elevada, variando de 80 a $85 \%$. Quando se compara o IS entre as diferentes épocas do ano, tem-se valores de 64 a 90\%. Tem-se, com isso, confirmada a homogeneidade na distribuição dos FMA tanto no que se refere ao tipo de vegetação, à época do ano e aos demais parâmetros abióticos.

Como conclusões, reafirma-se que a diversidade de FMA foi de 21 táxons, não havendo indicativos de seleção mediada pelo tipo de vegetação (eucalipto, transição e mata) e época do ano em que se realizaram as coletas (março, junho e janeiro); a diversidade de espécies de FMA em áreas tropicais é grande, sendo necessários estudos de levantamento, uma vez que é freqüente a observação de espécies ainda não descritas, como pode-se verificar neste trabalho.

\section{Agradecimentos}

Ao Instituto Agronômico de Campinas, pelas análises dos solos.

\section{Referências}

Abbott, L. K. \& Gazey, C. 1994. An ecological view of the formation of VA mycorrhizas. Plant and Soil 159: $69-78$.

Begovacz, A. 1995. Levantamento florístico preliminar das sub-classes Magnoliidae, Hamamelidae, Caryophyliidae, Dilleniidae, Rosidae (Sensu Cronquist 1981) na Ilha dos Eucaliptos da represa do Guarapiranga, São Paulo, SP. Monografia de conclusão de curso. Departamento de Biologia da Universidade de Santo Amaro, São Paulo.

Bononi, V. L. R. \& Trufem, S. F. B. 1983. Endomicorrizas vesículo-arbusculares da Reserva Biológica de Moji-Guaçu. Rickia 10: 55-84.

Bononi, V. L. R.; Barbosa, L. M. \& Viriato, A. 1988. Micorrizas vesículo-arbusculares em amendoim e em plantas invasoras de culturas. Hoehnea 15: 1-19.

Carrenho, R. 1993. Efeitos dos fungicidas sistêmicos fosetil-Al e metalaxil-mancozeb sobre as populações nativas de fungos micorrízicos arbusculares e sobre o estabelecimento natural da associação micorrízica em mudas cítricas (combinação varietal Citrus sinensis Osbeck Citrus limonia L.). Dissertação de Mestrado. UNESP, Rio Claro.

Cooper, K. M. \& Tinker, P.M. 1978. Translocation and transfer of nutrients in vesicular arbuscular mycorrhizas. New Phytologist 81: 43-53. 
Daniels, B. A. \& Skipper, H. D. 1982. Methods for the recovery and quantitative estimation of propagules from soil. Pp. 29-35. In: N. C. Schenck (Ed.), Methods and principles of mycorrhizal research. The Americam Phytopathological Society, St. Paul.

Francis, R.; Finlay, R. D. \& Read, D. J. 1986. Vesicular arbuscular mycorrhizas in natural vegetation systems. IV. Transfer of nutrients in inter- and intra-specific combinations of host plants. New Phytologist 102: 103-111.

Gerdemann, J. W. \& Nicolson, T. H. 1963. Spores of mycorrhizal Endogone species extracted from soil by wet sieving and decanting. Transactions of the British Mycological Society 46: 235-244.

Grandi, R. A. P. \& Trufem, S. F. B. 1991. Fungos micorrízicos vesículo-arbusculares em Marantaceae cultivadas no Instituto de Botânica, São Paulo, SP. Revista Brasileira de Botânica 14(2): 89-95.

Gomes, S. P. 1997. Fungos micorrízicos arbusculares da Ilha dos Eucaplitos, Represa do Guarapiranga, São Paulo, SP. Monografia de conclusão de curso. Departamento de Biologia da Universidade de Santo Amaro, São Paulo.

Lima, W. P. 1993. Impacto Ambiental do Eucalipto. 2ª edição. Editora da USP, São Paulo.

Malavolta, E. 1980. Elementos de nutrição mineral de plantas. Editora Agronômica Ceres Ltda., São Paulo.

Melo, A. A. 1994. Estudo qualitativo da fauna de Lepidopteras (borboletas) da bacia do Guarapiranga. Monografia de conclusão de curso. Departamento de Biologia da Faculdade de Santo Amaro, São Paulo.

Morton, J. B. \& Benny, G. L. 1990. Revised classification of arbuscular mycorrhizal fungi (Zygomycetes): A new order, Glomales, two new suborders, Glomineae e Gigasporineae, and two new families, Acaulosporaceae and Gigasporaceae, with an emendation of Glomales. Mycotaxon 37: 471-491.

Mosse, B; Stribley, D. P. \& Le Tacon, F. 1981. Ecology of mycorrhizae and mycorrhizal fungi. Advances in Microbial Ecology 5: 137-210.

Muller-Dombois, D. \& Ellemberg, H. 1974. Aims and methods of vegetatiom ecology. John Wiley \& Sons, New York.

Priorities for microbial biodiversity research. 1995. Summary and Recommendations. Workshop. Center for Microbial Ecology. Michigan State University.

Schenck, N. C. \& Pérez, Y. 1988. Manual for the identification of VA mycorrhizal fungi. INVAM. University of Florida, Gainesville.

Siqueira, J. O. \& Franco, A. A. 1988. Biotecnologia de Solo. MEC Ministério da Educação. ABEAS, Lavras. ESAL/FAEP, Brasília.

Trufem, S. F. B. 1988. Fungos micorrízicos vesículo-arbusculares da Ilha do Cardoso, SP, Brasil. Tese de Doutorado. Universidade de São Paulo, São Paulo.

Trufem, S. F. B. \& Grandi, R. A. P. \& Silveira, R. B. A. 1990. Fungos vesículo-arbusculares em plantas ornamentais do Jardim Botânico de São Paulo, SP. Hoehnea 17(1): 85-89.

Trufem, S. F. B. \& Viriato, A. 1990. Fungos micorrízicos-arbusculares da Reserva Biológica do Alto da Serra de Paranapiacaba, São Paulo, Brasil. Revista Brasileira de Botânica 13(1): 49-54.

Trufem, S. F. B. \& Malatinszky, S. M. M. 1995. Fungos micorrízicos arbusculares em Melastomataceae e outras plantas nativas resistentes e sensíveis à poluição na Reserva Biólogica do Alto da Serra de Paranapiacaba, SP, Brasil. Hoehnea 22(1/2): 77-89.

Wellbaum, C. 1996. Estudo preliminar dos fungos filamentosos em folhas do ambiente terrestre e aquático da Ilha dos Eucaliptos, Represa do Guarapiranga, Município de São Paulo, SP. Monografia de conclusão de curso. Departamento de Biologia da Universidade de Santo Amaro, São Paulo.

Zambolim, L. \& Siqueira, J. O. 1985. Importância e potencial das associações micorrízicas para a agricultura. EPAMIG, Belo Horizonte. 